\title{
Extending the Convergence of Two Similar Sixth Order Schemes for Solving Equations under Generalized Conditions
}

\author{
Ioannis K. Argyros ${ }^{1 *}$, Santhosh George $^{2}{ }^{\circledR}$, Christopher I. Argyros $^{3}$ \\ ${ }^{1}$ Department of Mathematical Sciences, Cameron University, Lawton, OK 73505, USA \\ ${ }^{2}$ Department of Mathematical and Computational Sciences, National Institute of Technology Karnataka, India \\ ${ }^{3}$ Department of Computing and Technology, Cameron University, OK 73505, USA \\ E-mail: iargyros@cameron.edu
}

Received: 10 June 2021; Revised: 16 August 2021; Accepted: 18 August 2021

\begin{abstract}
The applicability of two competing efficient sixth convergence order schemes is extended for solving Banach space valued equations. In previous works, the seventh derivative has been used not appearing on the schemes. But we use only the first derivative that appears on the scheme. Moreover, bounds on the error distances and results on the uniqueness of the solution are provided not given in the earlier works based on $\omega$-continuity conditions. Our technique extends other schemes analogously, since it is so general. Numerical examples complete this work.
\end{abstract}

Keywords: seventh convergence order, $\omega$-continuity, local convergence, Banach space

AMS: 65H10, 65G99, 49M15

\section{Introduction}

In [1], a multistep class of iterative methods is considered for approximating a solution $x_{*}$ of the equation

$$
\mathcal{G}(x)=0
$$

where $\mathcal{G}: \Omega \subseteq B_{1} \rightarrow B_{2}$ is nonlinear operator between the Banach spaces $B_{1}, B_{2}$ and $\Omega$ is a nonempty and open set. The solution $x_{*}$ is sought in closed form. But this is achieved only on special occasions. That is why iterative schemes are developed generating sequences converging to $x_{*}$ under suitable convergence criteria [2-6]. By $\mathcal{G}^{\prime}$ we denote the Frećhet derivative of operator $\mathcal{G}$ (see Definition 2.1 that follows).

We are concerned with the following three-step schemes developed for $x_{0} \in \Omega$ and all $n=0,1,2, \ldots$ by

$$
y_{n}=x_{n}-\frac{2}{3} \mathcal{G}^{\prime}\left(x_{n}\right)^{-1} \mathcal{G}\left(x_{n}\right)
$$

Copyright (C2021 Ioannis K. Argyros, et al.

DOI: https://doi.org/10.37256/cm.242021991

This is an open-access article distributed under a CC BY license

(Creative Commons Attribution 4.0 International License)

https://creativecommons.org/licenses/by/4.0/ 


$$
\begin{aligned}
& z_{n}=x_{n}-\frac{1}{2}\left(3 \mathcal{G}^{\prime}\left(y_{n}\right)-\mathcal{G}^{\prime}\left(x_{n}\right)\right)^{-1} \times\left(3 \mathcal{G}^{\prime}\left(y_{n}\right)+\mathcal{G}^{\prime}\left(x_{n}\right)\right) \mathcal{G}^{\prime}\left(x_{n}\right)^{-1} \mathcal{G}\left(x_{n}\right) \\
& x_{n+1}=z_{n}-\left(\frac{1}{2}\left(3 \mathcal{G}^{\prime}\left(y_{n}\right)-\mathcal{G}^{\prime}\left(x_{n}\right)\right)^{-1} \times\left(3 \mathcal{G}^{\prime}\left(y_{n}\right)+\mathcal{G}^{\prime}\left(x_{n}\right)\right)\right)^{2} \mathcal{G}^{\prime}\left(x_{n}\right)^{-1} \mathcal{G}\left(z_{n}\right)
\end{aligned}
$$

and

$$
\begin{aligned}
& y_{n}=x_{n}-\frac{2}{3} \mathcal{G}^{\prime}\left(x_{n}\right)^{-1} \mathcal{G}\left(x_{n}\right) \\
& z_{n}=x_{n}-\frac{1}{2}\left(3 \mathcal{G}^{\prime}\left(y_{n}\right)-\mathcal{G}^{\prime}\left(x_{n}\right)\right)^{-1} \times\left(3 \mathcal{G}^{\prime}\left(y_{n}\right)+\mathcal{G}^{\prime}\left(x_{n}\right)\right) \mathcal{G}^{\prime}\left(x_{n}\right)^{-1} \mathcal{G}\left(x_{n}\right) \\
& x_{n+1}=z_{n}-2\left(3 \mathcal{G}^{\prime}\left(y_{n}\right)-\mathcal{G}^{\prime}\left(x_{n}\right)\right)^{-1} \mathcal{G}\left(z_{n}\right) .
\end{aligned}
$$

The sixth convergence order of scheme (2) and scheme (3) is shown in [1, 7], respectively using Taylor series expansions and hypotheses up to the seventh derivative of $\mathcal{G}$ not appearing on these schemes. These hypotheses limit the applicability of scheme (2). As a motivational example, consider function. Let $f:\left[-\frac{1}{2}, \frac{3}{2}\right] \rightarrow \mathbb{R}$ defined by

$$
f(t)=\left\{\begin{array}{cc}
t^{3} \log t^{2}+t^{5}-t^{4} & \text { if } t \neq 0 \\
0 & \text { if } t=0
\end{array}\right.
$$

Then, we obtain $f^{\prime \prime \prime}(\mathrm{t})=6 \log t^{2}+60 t^{2}-24 t+22$. So, function $f^{\prime \prime \prime}$ is not bounded on $\left[-\frac{1}{2}, \frac{3}{2}\right]$. Hence, the results in [7] cannot be used to solve equation (1) using scheme (2) or scheme (3). Moreover, no upper bounds on $\left\|x_{n}-x_{*}\right\|$ or results on the uniqueness of $x_{*}$ were presented either. In this article, we develop a technique using only the derivatives of order one (that appears on the schemes) and provide upper bounds on $\left\|x_{n}-x_{*}\right\|$ and uniqueness results. This way, we extend the applicability of these schemes. We have used, the Computational Order of Convergence (COC) and Approximate Computational Order of Convergence (ACOC) to determine the convergence order which does not require the usage of higher-order derivatives or divided differences (see Remark 2.2) [8-12]. This is done in Section 2 and Section 3. Numerical examples appear in Section 4. This technique can be used on other methods [13-16].

Similar work has been done in [6]. But the relations do not imply each other and are hard to determine the ones in this paper based on [6].

\section{Convergence}

Some standard definitions and results are restated in order to make the paper as self-contained as possible. More on this topic can be found in $[4,9-11,17]$. The set $L(X, Y)$ denotes the space of bounded linear operators from $X$ into $Y$.

Definition 2.1 The operator $F: \Omega \subset X \rightarrow Y$ is Fréchet-differentiable at $x \in \Omega$ if there exists an $A \in L(X, Y)$ such that

$$
\lim _{h \rightarrow 0} \frac{1}{\|h\|}\|\mathcal{G}(x+h)-\mathcal{G}(x)-A(x)\|=0 .
$$


The linear operator $A$ is denoted by $\mathcal{G}^{\prime}(x)$ and is called the Fréchet derivative of $F$ at $x$.

Next, we present the celebrated Banach Lemma on invertible operators.

Lemma 2.2 Let $A, B \in L(X, Y)$ with $A^{-1} \in L(Y, X)$ and $\left\|A^{-1}\right\|\|A-B\|<1$. Then $B^{-1} \in L(Y, X)$ and

$$
\left\|B^{-1}\right\| \leq \frac{\left\|A^{-1}\right\|}{1-\left\|A^{-1}\right\|\|A-B\|}
$$

Next, we develop parameters and scalar functions. Let $M=[0, \infty)$.

Assume equation:

(i)

$$
\varsigma_{0}(t)-1=0
$$

has a least positive solution $\rho_{0}$ for some function $\varsigma_{0}: M \rightarrow M$ nondecreasing and continuous. Let $M_{0}=\left[0, \rho_{0}\right)$.

(ii)

$$
h_{1}(t)-1=0
$$

has a least solution $R_{1} \in\left(0, \rho_{0}\right)$ for functions $\varsigma: M_{0} \rightarrow M, \varsigma_{1}: M_{0} \rightarrow M$ nondecreasing and continuous such that for

$$
h_{1}(t)=\frac{\int_{0}^{1} \varsigma((1-\tau) t) d \tau+\frac{1}{3} \int_{0}^{1} \varsigma_{1}(\theta t) d \theta}{1-\varsigma_{0}(t)}
$$

(iii)

$$
1-p(t)=0
$$

has a least solution $\rho_{p} \in\left(0, \rho_{0}\right)$ for $p(t)=\frac{1}{2}\left(3 \varsigma_{0}\left(h_{1}(t) t\right)+\varsigma_{0}(t)\right)$. Set $M_{p}=\left[0, \rho_{\mathrm{p}}\right)$.

(iv)

$$
h_{2}(t)=0 \text { and } \varsigma_{0}\left(h_{2}(t) t\right)-1=0
$$

has least solutions $R_{2}, \rho_{1} \in\left(0, \rho_{p}\right)$, respectively for functions $h_{2}: M_{p} \rightarrow M$ defined by

$$
h_{2}(t)=\frac{\int_{0}^{1} \varsigma((1-\theta) t) d \theta}{1-\varsigma_{0}(t)}+\frac{3\left(\varsigma_{0}\left(h_{1}(t) t\right)+\varsigma_{0}(t)\right) \int_{0}^{1} \varsigma_{1}(\theta t) d \theta}{4\left(1-\varsigma_{0}(t)\right)(1-p(t))} .
$$

Let $\rho=\min \left\{\rho_{0}, \rho_{1}\right\}$ and $M_{1}=[0, \rho)$.

(v)

$$
h_{3}(t)-1=0,
$$

has a least solution $R_{3} \in(0, \rho)$, where 


$$
\begin{aligned}
h_{3}(t)= & {\left[\frac{\int_{0}^{1} \varsigma\left((1-\theta) h_{2}(t) t\right) d \theta}{1-\varsigma_{0}\left(h_{2}(t) t\right)}+\frac{\left(\varsigma_{0}\left(h_{2}(t) t\right)+\varsigma_{0}(t)\right) \int_{0}^{1} \varsigma_{1}\left(\theta h_{2}(t) t\right) d \theta}{\left(1-\varsigma_{0}\left(h_{2}(t) t\right)\right)\left(1-\varsigma_{0}(t)\right)}\right.} \\
& \left.+\frac{3}{4}\left(\frac{3 \varsigma_{0}\left(h_{1}(t) t\right)+\varsigma_{0}(t)}{(1-p(t)}\right) \times\left(1+\frac{3\left(3 \varsigma_{1}\left(h_{1}(t) t\right)+\varsigma_{1}(t)\right)}{4(1-p(t))}\right)\right] \frac{\int_{0}^{1} \varsigma_{1}\left(\theta h_{2}(t) t\right) d \theta}{1-\varsigma_{0}(t)}
\end{aligned}
$$

Notice that the preceding multiplications are well defined as products between real functions.

We shall show that

$$
R=\min \left\{R_{j}\right\}, j=1,2,3
$$

is a radius of convergence for scheme (2). By these definitions, we have that for each $t \in[0, R)$

$$
\begin{gathered}
0 \leq \varsigma_{0}(t)<1 \\
0 \leq p(t)<1,0 \leq \xi_{0}\left(h_{2}(t) t\right)<0
\end{gathered}
$$

and

$$
0 \leq h_{j}(t)<1
$$

Moreover, define $B\left(x_{*}, \varepsilon\right)=\left\{x \in X:\left\|x-x_{*}\right\|<\varepsilon\right\}$ and $\bar{B}\left(x_{*}, \varepsilon\right)=\left\{x \in X:\left\|x-x_{*}\right\| \leq \varepsilon\right\}, \varepsilon>0$.

The conditions $(\mathcal{A})$ shall be used in the local convergence analysis of scheme (2) that follows.

$\left(\mathcal{A}_{1}\right)$ There exists a simple solution $x_{*}$ of equation (4)

$\left(\mathcal{A}_{2}\right)$ There exists function $\varsigma_{0}: M \rightarrow M$ nondecreasing and continuous such that for all $x \in \Omega$

$$
\left\|\mathcal{G}^{\prime}\left(x_{*}\right)^{-1}\left(\mathcal{G}^{\prime}(x)-\mathcal{G}^{\prime}\left(x_{*}\right)\right)\right\| \leq \varsigma_{0}\left(\left\|x-x_{*}\right\|\right) \text {. }
$$

Set $\Omega_{0}=\Omega \cap B\left(x_{*}, \rho_{0}\right)$.

$\left(\mathcal{A}_{3}\right)$ There exists function $\varsigma: M_{0} \rightarrow M, \varsigma_{1}: M_{0} \rightarrow M$ nondecreasing and continuous such that for each $x, y \in \Omega_{0}$

$$
\left\|\mathcal{G}^{\prime}\left(x_{*}\right)^{-1}\left(\mathcal{G}^{\prime}(x)-\mathcal{G}^{\prime}(y)\right)\right\| \leq \varsigma(\|x-y\|)
$$

and

$$
\left\|\mathcal{G}^{\prime}\left(x_{*}\right)^{-1} \mathcal{G}^{\prime}(x)\right\| \leq \varsigma_{1}\left(\left\|x-x_{*}\right\|\right)
$$

$\left(\mathcal{A}_{4}\right) \bar{B}\left(x_{*}, \tilde{R}\right) \subseteq \Omega$, where $\tilde{R}$ to be determined later, and

$\left(\mathcal{A}_{5}\right)$ There exists $\rho_{*} \geq R$ such that

$$
\int_{0}^{1} \varsigma_{0}\left(\rho_{*} \theta\right) d \theta<1
$$

Set $\Omega_{1}=\Omega \cap \bar{B}\left(x_{*}, \rho\right)$. 
Next, based on the conditions $(\mathcal{A})$ and the preceding notation, we show the main local convergence analysis for scheme (2).

Theorem 2.3 Assume conditions $(\mathcal{A})$ hold with $\tilde{R}=R$, and starter $x_{0} \in B\left(x_{*}, R\right)-\left\{x_{*}\right\}$. Then, sequence $\left\{x_{n}\right\}$ developed by scheme (2) is well defined in $B\left(x_{*}, R\right)$, remains in $B\left(x_{*}, R\right)$ and converges to $x_{*}$. Moreover, $x_{*}$ is the only solution of equation (4) in the set $\Omega_{1}$ given in $\left(\mathcal{A}_{5}\right)$

Proof. Let $e_{n}=\left\|x_{n}-x_{*}\right\|$. Choose $u \in B\left(x_{*}, R\right)-\left\{x_{*}\right\}$. By using $\left(\mathcal{A}_{2}\right),(9)$ and (10)

$$
\left\|\mathcal{G}^{\prime}\left(x_{*}\right)^{-1}\left(\mathcal{G}^{\prime}(u)-\mathcal{G}^{\prime}\left(x_{*}\right)\right)\right\| \leq \varsigma_{0}\left(\left\|u-x_{*}\right\|\right) \leq \varsigma_{0}(R)<1
$$

which together with the Banach lemma on invertible operators given in Lemma 2.2 and scheme (2) give

$$
\left\|\mathcal{G}^{\prime}(u)^{-1} \mathcal{G}^{\prime}\left(x_{*}\right)\right\| \leq \frac{1}{1-\varsigma_{0}\left(\left\|u-x_{*}\right\|\right)}
$$

and that $y_{0}$ exists. So, we can write by the first substep of scheme (2) for $n=0$ and $\left(\mathcal{A}_{1}\right)$ that

$$
y_{0}-x_{*}=x_{0}-x_{*}-\mathcal{G}^{\prime}\left(x_{0}\right)^{-1} \mathcal{G}\left(x_{0}\right)+\frac{1}{2} \mathcal{G}^{\prime}\left(x_{0}\right)^{-1} \mathcal{G}\left(x_{0}\right)
$$

Then, in view of (9), (12) (for $j=1),\left(\mathcal{A}_{3}\right),(13)$ (for $\left.u=x_{0}\right),(14)$, and the triangle inequality, we have in turn

$$
\begin{aligned}
\left\|y_{0}-x_{*}\right\| & \leq\left\|x_{0}-x_{*}-\mathcal{G}^{\prime}\left(x_{0}\right)^{-1} \mathcal{G}\left(x_{0}\right)\right\|+\frac{1}{3}\left\|\mathcal{G}^{\prime}\left(x_{0}\right)^{-1} \mathcal{G}^{\prime}\left(x_{*}\right)\right\|\left\|\mathcal{G}^{\prime}\left(x_{*}\right)^{-1} \mathcal{G}\left(x_{0}\right)\right\| \\
& \leq\left\|\mathcal{G}^{\prime}\left(x_{0}\right)^{-1} \mathcal{G}^{\prime}\left(x_{*}\right)\right\| \times \int_{0}^{1}\left\|\mathcal{G}^{\prime}\left(x_{*}\right)^{-1}\left(\mathcal{G}^{\prime}\left(x_{*}+\theta\left(x_{0}-x_{*}\right)\right)-\mathcal{G}^{\prime}\left(x_{0}\right)\right)\right\| d \theta e_{0} \\
& \leq \frac{\int_{0}^{1} \varsigma\left((1-\theta) e_{0}\right) d \theta+\frac{1}{3} \int_{0}^{1} \varsigma_{1}\left(\theta e_{0}\right) d \theta}{1-\varsigma_{0}\left(e_{0}\right)} e_{0} \\
& \leq h_{1}\left(e_{0}\right) e_{0} \\
& \leq e_{0}<R
\end{aligned}
$$

so $y_{0} \in B\left(x_{*}, R\right)$.

Next, we show $\left(3 \mathcal{G}^{\prime}\left(y_{0}\right)-\mathcal{G}^{\prime}\left(x_{0}\right)\right)$ is invertible. Using $\left(\mathcal{A}_{2}\right),(9),(11)$ and $(15)$, we get in turn that

$$
\begin{aligned}
& \left\|\left(2 \mathcal{G}^{\prime}\left(x_{*}\right)\right)^{-1}\left(3 \mathcal{G}^{\prime}\left(y_{0}\right)-\mathcal{G}^{\prime}\left(x_{0}\right)-2 \mathcal{G}^{\prime}\left(x_{*}\right)\right)\right\| \\
\leq & \frac{1}{2}\left[3\left\|\mathcal{G}^{\prime}\left(x_{*}\right)^{-1}\left(\mathcal{G}^{\prime}\left(y_{0}\right)-\mathcal{G}^{\prime}\left(x_{*}\right)\right)\right\|+\left\|\mathcal{G}^{\prime}\left(x_{*}\right)^{-1}\left(\mathcal{G}^{\prime}\left(x_{0}\right)-\mathcal{G}^{\prime}\left(x_{*}\right)\right)\right\|\right] \\
\leq & \frac{1}{2}\left(3 \varsigma_{0}\left(\left\|y_{0}-x_{*}\right\|\right)+\varsigma_{0}\left(e_{0}\right)\right) \leq p\left(e_{0}\right) \leq p(R)<1,
\end{aligned}
$$


so, again by Lemma 2.2

$$
\left\|\left(3 \mathcal{G}^{\prime}\left(y_{0}\right)-\mathcal{G}^{\prime}\left(x_{0}\right)\right)^{-1} \mathcal{G}^{\prime}\left(x_{*}\right)\right\| \leq \frac{1}{2\left(1-p\left(e_{0}\right)\right)}
$$

and $z_{0}, x_{1}$ exist. Then, by (9), (12) (for $j=2,3$ ), (13) (for $u=x_{0}$ ), (15), and (16), we obtain in turn

$$
\begin{aligned}
\left\|z_{0}-x_{*}\right\|= & \left\|x_{0}-x_{*}-\mathcal{G}^{\prime}\left(x_{0}\right)^{-1} \mathcal{G}\left(x_{0}\right)\left[I-\frac{1}{2}\left(3 \mathcal{G}^{\prime}\left(y_{0}\right)-\mathcal{G}^{\prime}\left(x_{0}\right)\right)^{-1}\left(3 \mathcal{G}^{\prime}\left(y_{0}\right)+\mathcal{G}^{\prime}\left(x_{0}\right)\right)\right] \mathcal{G}^{\prime}\left(x_{0}\right)^{-1} \mathcal{G}\left(x_{0}\right)\right\| \\
& \leq\left[\frac{\int_{0}^{1} \zeta\left((1-\theta) e_{0}\right) d \theta}{1-\varsigma_{0}\left(e_{0}\right)}+\frac{3}{4} \frac{\left(\varsigma_{0}\left(\left\|y_{0}-x_{*}\right\|\right)+\varsigma_{0}\left(e_{0}\right)\right) \int_{0}^{1} \varsigma_{1}\left(\theta e_{0}\right) d \theta}{\left(1-\varsigma_{0}\left(e_{0}\right)\right)\left(1-p\left(e_{0}\right)\right)}\right] e_{0} \\
& \leq h_{2}\left(e_{0}\right) e_{0} \leq e_{0},
\end{aligned}
$$

and

$$
\begin{aligned}
\left\|x_{1}-x_{*}\right\| \leq & \| z_{0}-x_{*}-\mathcal{G}^{\prime}\left(z_{0}\right)^{-1} \mathcal{G}\left(z_{0}\right)+\mathcal{G}^{\prime}\left(z_{0}\right)^{-1}\left(\mathcal{G}^{\prime}\left(x_{0}\right)-\mathcal{G}^{\prime}\left(z_{0}\right)\right) \mathcal{G}^{\prime}\left(x_{0}\right)^{-1} \mathcal{G}\left(z_{0}\right) \\
& +\left(I-\left(\frac{1}{2}\left(3 \mathcal{G}^{\prime}\left(y_{0}\right)-\mathcal{G}^{\prime}\left(x_{0}\right)\right)^{-1}\left(3 \mathcal{G}^{\prime}\left(y_{0}\right)+\mathcal{G}^{\prime}\left(x_{0}\right)\right)\right)^{2}\right) \mathcal{G}^{\prime}\left(x_{0}\right)^{-1} \mathcal{G}\left(z_{0}\right) \| \\
\leq & {\left[\frac{\int_{0}^{1} \varsigma\left((1-\theta)\left\|z_{0}-x_{*}\right\|\right) d \theta}{1-\varsigma_{0}\left(\left\|z_{0}-x_{*}\right\|\right)}+\frac{\left(\varsigma_{0}\left(\left\|z_{0}-x_{*}\right\|\right)+\varsigma_{0}\left(e_{0}\right)\right) \int_{0}^{1} \varsigma_{1}\left(\theta\left\|z_{0}-x_{*}\right\|\right) d \theta}{\left(1-\varsigma_{0}\left(\left\|z_{0}-x_{*}\right\|\right)\right)\left(1-\varsigma_{0}\left(e_{0}\right)\right)}\right.} \\
& +\left(\frac{3\left(3 \varsigma_{0}\left(\left\|y_{0}-x_{*}\right\|\right)+\varsigma_{0}\left(e_{0}\right)\right.}{4\left(1-p\left(e_{0}\right)\right)}\right) \times\left(1+\frac{3\left(3 \varsigma_{0}\left(\left\|y_{0}-x_{*}\right\|\right)+\varsigma_{1}\left(e_{0}\right)\right.}{4\left(1-p\left(e_{0}\right)\right)}\right) \\
& \left.+\frac{\int_{0}^{1} \varsigma_{1}\left(\theta\left\|z_{0}-x_{*}\right\|\right) d \theta}{1-\varsigma_{0}\left(e_{0}\right)}\right]\left\|z_{0}-x_{*}\right\| \\
\leq & h_{3}\left(e_{0}\right) e_{0} \leq e_{0},
\end{aligned}
$$

so, $z_{0}, x_{1} \in B\left(x_{*}, R\right)$, where we also used that the large parenthesis can be written as

$$
\left(I-\left(\frac{1}{2}\left(3 \mathcal{G}^{\prime}\left(y_{0}\right)-\mathcal{G}^{\prime}\left(x_{0}\right)\right)^{-1}\left(3 \mathcal{G}^{\prime}\left(y_{0}\right)+\mathcal{G}^{\prime}\left(x_{0}\right)\right)\right)\right) \times\left(I+\left(\frac{1}{2}\left(3 \mathcal{G}^{\prime}\left(y_{0}\right)-\mathcal{G}^{\prime}\left(x_{0}\right)\right)^{-1}\left(3 \mathcal{G}^{\prime}\left(y_{0}\right)+\mathcal{G}^{\prime}\left(x_{0}\right)\right)\right)\right)
$$

and 


$$
\begin{aligned}
& {\left[I-\frac{1}{2}\left(3 \mathcal{G}^{\prime}\left(y_{0}\right)-\mathcal{G}^{\prime}\left(x_{0}\right)\right)^{-1}\left(3 \mathcal{G}^{\prime}\left(y_{0}\right)+\mathcal{G}^{\prime}\left(x_{0}\right)\right)\right] } \\
&=\left(3 \mathcal{G}^{\prime}\left(y_{0}\right)-\mathcal{G}^{\prime}\left(x_{0}\right)\right)^{-1}\left[\left(3 \mathcal{G}^{\prime}\left(y_{0}\right)-\mathcal{G}^{\prime}\left(x_{0}\right)\right)-\frac{1}{2}\left(3 \mathcal{G}^{\prime}\left(y_{0}\right)+\mathcal{G}^{\prime}\left(x_{0}\right)\right)\right] \\
&= \frac{3}{2}\left(3 \mathcal{G}^{\prime}\left(y_{0}\right)-\mathcal{G}^{\prime}\left(x_{0}\right)\right)^{-1}\left(\mathcal{G}^{\prime}\left(y_{0}\right)-\mathcal{G}^{\prime}\left(x_{0}\right)\right) . \\
&\left(I-\left(\frac{1}{2}\left(3 \mathcal{G}^{\prime}\left(y_{0}\right)-\mathcal{G}^{\prime}\left(x_{0}\right)\right)^{-1}\left(3 \mathcal{G}^{\prime}\left(y_{0}\right)+\mathcal{G}^{\prime}\left(x_{0}\right)\right)\right)\right) \\
&=\left.\frac{1}{2}\left(3 \mathcal{G}^{\prime}\left(y_{0}\right)-\mathcal{G}^{\prime}\left(x_{0}\right)\right)^{-1}\left(6 \mathcal{G}^{\prime}\left(y_{0}\right)-2 \mathcal{G}^{\prime}\left(x_{0}\right)-3 \mathcal{G}^{\prime}\left(y_{0}\right)-\mathcal{G}^{\prime}\left(x_{0}\right)\right)\right) \\
&= \frac{3}{2}\left(3 \mathcal{G}^{\prime}\left(y_{0}\right)-\mathcal{G}^{\prime}\left(x_{0}\right)\right)^{-1}\left(\mathcal{G}^{\prime}\left(y_{0}\right)-\mathcal{G}^{\prime}\left(x_{0}\right)\right), \\
&\left\|\left(I-\left(\frac{1}{2}\left(3 \mathcal{G}^{\prime}\left(y_{0}\right)-\mathcal{G}^{\prime}\left(x_{0}\right)\right)^{-1}\left(3 \mathcal{G}^{\prime}\left(y_{0}\right)+\mathcal{G}^{\prime}\left(x_{0}\right)\right)\right)\right)\right\| \leq \frac{3}{4} \frac{3 \varsigma_{0}\left(\left\|y_{0}-x_{*}\right\|\right)+\varsigma_{0}\left(e_{0}\right)}{1-p\left(e_{0}\right)} \\
&\left\|\left(I+\left(\frac{1}{2}\left(3 \mathcal{G}^{\prime}\left(y_{0}\right)-\mathcal{G}^{\prime}\left(x_{0}\right)\right)^{-1}\left(3 \mathcal{G}^{\prime}\left(y_{0}\right)+\mathcal{G}^{\prime}\left(x_{0}\right)\right)\right)\right)\right\| \leq 1+\frac{3}{4} \frac{3 \varsigma_{0}\left(\left\|y_{0}-x_{*}\right\|\right)+\varsigma_{0}\left(e_{0}\right)}{1-p\left(e_{0}\right)} .
\end{aligned}
$$

Hence, so far we have shown estimates (15), (17) and (18), for $n=0$ and $y_{0}, z_{0}, x_{1} \in B\left(x_{*}, R\right)$. Next, we suppose estimates

$$
\begin{gathered}
\left\|y_{m}-x_{*}\right\| \leq h_{1}\left(e_{m}\right) e_{m}, \\
\left\|z_{m}-x_{*}\right\| \leq h_{2}\left(e_{m}\right) e_{m}
\end{gathered}
$$

and

$$
\left\|x_{m+1}-x_{*}\right\| \leq h_{3}\left(e_{m}\right) e_{m}
$$

hold for $m=0,1,2, \ldots, n$. We shall show that they hold for $m=n+1$. Then, by repeating these calculations with $x_{0}, y_{0}$, $z_{0}, x_{1}$ replaced by $x_{m}, y_{m}, z_{m}, x_{m+1}$ respectively, we complete the induction and get

$$
\left\|x_{m+1}-x_{*}\right\| \leq \gamma\left\|x_{m}-x_{*}\right\|<R
$$

where $\gamma=h_{3}\left(e_{0}\right) \in[0,1)$, so $\lim _{m \rightarrow \infty} x_{m}=x_{*}$ and $x_{m+1} \in B\left(x_{*}, R\right)$.

Finally, let $q \in \Omega_{1}$ with $\mathcal{G}(q)=0$. Consider $S=\int_{0}^{1} \mathcal{G}^{\prime}\left(x_{*}+\theta\left(q-x_{*}\right)\right) d \theta$. It follows by $\left(\mathcal{A}_{2}\right)$ that 


$$
\left\|\mathcal{G}^{\prime}\left(x_{*}\right)^{-1}\left(S-\mathcal{G}^{\prime}\left(x_{*}\right)\right)\right\| \leq \int_{0}^{1} \varsigma_{0}\left(\theta\left\|x_{*}-q\right\|\right) d \theta \leq \int_{0}^{1} \varsigma_{0}(\theta \rho)<1,
$$

so $x_{*}=q$, since $S$ is invertibile and $0=\mathcal{G}(q)-\mathcal{G}\left(x_{*}\right)=S\left(q-x_{*}\right)$.

Remark 2.4 1. In view of $\left(\mathcal{A}_{2}\right)$ and the estimate

$$
\begin{aligned}
\left\|F^{\prime}\left(x^{*}\right)^{-1} F^{\prime}(x)\right\| & =\left\|F^{\prime}\left(x^{*}\right)^{-1}\left(F^{\prime}(x)-F^{\prime}\left(x^{*}\right)\right)+I\right\| \\
& \leq 1+\left\|F^{\prime}\left(x^{*}\right)^{-1}\left(F^{\prime}(x)-F^{\prime}\left(x^{*}\right)\right)\right\| \leq 1+\varsigma_{0}\left(\left\|x-x^{*}\right\|\right)
\end{aligned}
$$

the second condition in $\left(\mathcal{A}_{3}\right)$ can be dropped and $\varsigma_{1}$ can be replaced by

$$
\varsigma_{1}(t)=1+\varsigma_{0}(t)
$$

or

$$
\varsigma_{1}(t)=1+\varsigma_{0}(R), \text { or } \varsigma_{1}(t)=2,
$$

since $t \in\left[0, \rho_{0}\right)$.

2. The results obtained here can be used for operators $F$ satisfying autonomous differential equations [3] of the form

$$
F^{\prime}(x)=P(F(x))
$$

where $P$ is a continuous operator. Then, since $F^{\prime}\left(x^{*}\right)=P\left(F\left(x^{*}\right)\right)=P(0)$, we can apply the results without actually knowing $x^{*}$. For example, let $F(x)=e^{x}-1$. Then, we can choose: $P(x)=x+1$.

3. Let $\varsigma_{0}(t)=L_{0} t$, and $\varsigma(t)=L t$. In [5], we showed that $r_{A}=\frac{2}{2 L_{0}+L}$ is the convergence radius of Newton's method:

$$
x_{n+1}=x_{n}-F^{\prime}\left(x_{n}\right)^{-1} F\left(x_{n}\right) \text { for each } n=0,1,2, \cdots
$$

under the conditions $\left(\mathcal{A}_{1}\right)-\left(\mathcal{A}_{3}\right)$. It follows from the definition of $r_{A}$, that the convergence radius $R$ of the method (2) cannot be larger than the convergence radius $r_{A}$ of the second order Newton's method (21). As already noted in [5] $r_{A}$ is at least as large as the convergence radius given by Rheinboldt [15]

$$
r_{R}=\frac{2}{3 L},
$$

where $L_{1}$ is the Lipschitz constant on $D$. The same value for $r_{R}$ was given by Traub [16]. In particular, for $L_{0}<L_{1}$ we have that

$$
r_{R}<r_{A}
$$

and 


$$
\frac{r_{R}}{r_{A}} \rightarrow \frac{1}{3} \text { as } \frac{L_{0}}{L_{1}} \rightarrow 0
$$

That is the radius of convergence $r_{A}$ is at most three times larger than Rheinboldt.

4. We can compute the computational order of convergence (COC) defined by

$$
\lambda=\ln \left(\frac{d_{n+1}}{d_{n}}\right) / \ln \left(\frac{d_{n}}{d_{n-1}}\right)
$$

or the approximate computational order of convergence

$$
\lambda_{1}=\ln \left(\frac{e_{n+1}}{e_{n}}\right) / \ln \left(\frac{e_{n}}{e_{n-1}}\right) .
$$

Next, we develop analogously the local convergence analysis of scheme (3).

But this time

$$
\bar{h}_{3}(t)=\left[\frac{\int_{0}^{1} \varsigma\left((1-\theta) h_{2}(t) t\right) d \theta}{1-\varsigma_{0}\left(h_{2}(t) t\right)}+\frac{\left(\varsigma_{0}(t)+3 \varsigma_{0}\left(h_{1}(t) t\right)+2 \varsigma_{0}\left(h_{2}(t) t\right)\right) \int_{0}^{1} \varsigma_{1}\left(\theta h_{2}(t) t\right) d \theta}{2\left(1-\varsigma_{0}(t) t\right)(1-p(t))}\right] h_{2}(t)
$$

Let $\bar{R}=\min \left\{R_{1}, R_{2}, \bar{R}_{3}\right\}$, where $\bar{R}_{3}$ is the least zero in $D_{2}-\{0\}$ of equation $\bar{h}_{3}(t)-1$ (assuming it exists). Denote by (A)' conditions (A) but $\tilde{R}=\bar{R}$. The definition of $\bar{h}_{3}$ is motivated by the estimate

$$
\begin{aligned}
\left\|x_{1}-x_{*}\right\| \leq & \| z_{0}-x_{*}-\mathcal{G}^{\prime}\left(z_{0}\right)^{-1} \mathcal{G}\left(z_{0}\right)+\mathcal{G}^{\prime}\left(z_{0}\right)^{-1}\left(3 \mathcal{G}^{\prime}\left(y_{0}\right)\right. \\
& \left.-2 \mathcal{G}^{\prime}\left(z_{0}\right)-\mathcal{G}^{\prime}\left(x_{0}\right)\right) \times\left(3 \mathcal{G}^{\prime}\left(y_{0}\right)-\mathcal{G}^{\prime}\left(x_{0}\right)^{-1} \mathcal{G}\left(z_{0}\right) \|\right. \\
\leq & \bar{h}_{3}\left(e_{0}\right) e_{0} \leq e_{0}<\bar{R} .
\end{aligned}
$$

Hence, we arrived at the corresponding local convergence result for scheme (3).

Theorem 2.5 Suppose conditions (A)' for $\tilde{R}=\bar{R}$ hold. Then, the conclusions of Theorem 2.3 hold for scheme (3) with $\bar{R}, \bar{h}_{3}$ replacing $R, h_{3}$, respectively.

Table 1. Radius for example 3.1

\begin{tabular}{ccc}
\hline Radius & $\varsigma_{1}(t)=1$ & $\varsigma_{1}(t)=1+\varsigma_{0}(t)$ \\
\hline$R_{1}$ & 0.44444 & 0.4 \\
$R_{2}$ & 0.277466 & 0.259201 \\
$R_{3}$ & 0.0938063 & 0.0896749 \\
$\bar{R}_{3}$ & 0.250116 & 0.234502 \\
\hline
\end{tabular}




\section{Numerical examples}

We use examples to find the radius of convergence for the schemes. Conditions $(\mathcal{A})$ are used and Definition 2.1 to determine the scalar functions. The radii are found by solving the " $h$ " equations.

Example 3.1 Let $B_{1}=B_{2}=\Omega=\mathbb{R}$. Define $\mathcal{G}(x)=\sin x$. Using conditions $(\mathcal{A})$ and Definition 2.1 we get $\mathcal{G}^{\prime}(x)=\cos$ $x$. Moreover, notice that $x_{*}=0$. By plugging these on conditions $\left(\mathcal{A}_{1}\right)-\left(\mathcal{A}_{3}\right)$, we see that they are satisfied provided we choose $\varsigma_{0}(t)=\varsigma(t)=t$ and $\varsigma_{1}(t)=1$. Then, by (9) we have the following radii (Table 1).

Example 3.2 Let $B_{1}=B_{2}=C[0,1]$, the space of continuous functions defined on $[0,1]$ with the max norm. Let $\Omega$ $=\bar{U}(0,1)$. Define function $F$ on $\Omega$ by

$$
\mathcal{G}(\psi)(x)=\psi(x)-5 \int_{0}^{1} x \theta \psi(\theta)^{3} d \theta
$$

We have by Definition 2.1 that

$$
\mathcal{G}^{\prime}(\psi(\mu))(x)=\mu(x)-15 \int_{0}^{1} x \theta \psi(\theta)^{2} \mu(\theta) d \theta, \text { for each } \mu \in D .
$$

Moreover, notice that $x_{*}=0$. By plugging these on conditions $\left(\mathcal{A}_{1}\right)-\left(\mathcal{A}_{3}\right)$, we see that they are satisfied provided that $\varsigma_{0}(t)=\varsigma_{1}(t)=\frac{15}{2} t, \varsigma_{1}(t)=2$. Then, by (9) we have the following radii (Table 2).

Table 2. Radius for example 3.2

\begin{tabular}{ccc}
\hline Radius & $\varsigma_{1}(t)=2$ & $\varsigma_{1}(t)=1+\varsigma_{0}(t)$ \\
\hline$R_{1}$ & 0.0296296 & 0.0727273 \\
$R_{2}$ & 0.0231907 & 0.0533333 \\
$R_{3}$ & 0.00330118 & 0.0119567 \\
$\bar{R}_{3}$ & 0.0199532 & 0.0312669 \\
\hline
\end{tabular}

Example 3.3 Let $B_{1}=B_{2}=\mathbb{R}^{3}, \Omega=U(0,1), x_{*}=(0,0,0)^{T}$, and define $F$ on $\Omega$ by

$$
\mathcal{G}(x)=\mathcal{G}\left(u_{1}, u_{2}, u_{3}\right)=\left(e^{u_{1}}-1, \frac{e-1}{2} u_{2}^{2}+u_{2}, u_{3}\right)^{T} .
$$

For the points $u=\left(u_{1}, u_{2}, u_{3}\right)^{T}$, the Fréchet derivative is given because of Definition 2.1 by

$$
\mathcal{G}^{\prime}(u)=\left(\begin{array}{ccc}
e^{u_{1}} & 0 & 0 \\
0 & (e-1) u_{2}+1 & 0 \\
0 & 0 & 1
\end{array}\right)
$$

Using the norm of the maximum of the rows and since $\mathcal{G}^{\prime}\left(x_{*}\right)=\operatorname{diag}(1,1,1)$, we get that conditions $(\mathcal{A})$ are verified if we choose $\varsigma_{0}(t)=(e-1) t, \varsigma(t)=e^{\frac{1}{e-1}} t$, and $\varsigma_{1}(t)=e^{\frac{1}{e-1}}$. Then, by (9) we have the following radii (Table 3). 
Table 3. Radius for example 3.3

\begin{tabular}{ccc}
\hline Radius & $\varsigma_{1}(t)=e^{\frac{1}{e-1}}$ & $\varsigma_{1}(t)=1+\varsigma_{0}(t)$ \\
\hline$R_{1}$ & 0.0154407 & 0.229929 \\
$R_{2}$ & 0.110108 & 0.149959 \\
$R_{3}$ & 0.0183481 & 0.0520623 \\
$\bar{R}_{3}$ & 0.0955553 & 0.206634 \\
\hline
\end{tabular}

Example 3.4 Returning back to the motivational example at the introduction of this study, we have for $x_{*}=1$ that condition $(\mathcal{A})$ are satisfied provided that we choose $\varsigma_{0}(t)=\varsigma(t)=96.662907 t, \varsigma_{1}(t)=1.0631$. Then, by (9) we have the following radii (Table 4$)$.

Table 4. Radius for example 3.4

\begin{tabular}{ccc}
\hline Radius & $\varsigma_{1}(t)=1.0631$ & $\varsigma_{1}(t)=1+\varsigma_{0}(t)$ \\
\hline$R_{1}$ & 0.00445282 & 0.00413809 \\
$R_{2}$ & 0.0104863 & 0.00268149 \\
$R_{3}$ & 0.000879059 & 0.0009277 \\
$\bar{R}_{3}$ & 0.00249313 & 0.0024257 \\
\hline
\end{tabular}

\section{References}

[1] Soleymani F, Lofti T, Bakhtiari P. A multi-step class of iterative methods for nonlinear systems. Optimization Letters. 2014; 8: 1001-1015.

[2] Amat S, Busquier S. Convergence and numerical analysis of two-step Steffensen's methods. Computers \& Mathematics with Applications. 2005; 49: 13-22.

[3] Amat S, Busquier S. A two-step Steffensen's under modified convergence conditions. Journal of Mathematical Analysis and Applications. 2006; 324: 1084-1092.

[4] Agarwal RP, Gala S, Ragusa MA. A regularity criterion in weak spaces to Boussinesq equations. Mathematics. 2020; 8(6): 920.

[5] Argyros IK. Computational theory of iterative methods. In: Chui CK, Wuytack L. (eds.) Studies in Computational Mathematics. Elsevier, New York, USA. 2007.

[6] George S, Argyros IK. Local comparison of two sixth order solvers using only the first derivative. Advances in the Theory of Nonlinear Analysis and its Application. 2019; 3(4): 220-230.

[7] Cordero A, Hueso JL, Martinez E, Torregrosa JR. A modified NewtonJarratt's composition. Numerical Algorithms. 2010; 55: 87-99.

[8] Argyros IK, Hilout S. Weaker conditions for the convergence of Newton's method. Journal of Complexity. 2012; 28: 364-387.

[9] Argyros IK, Magreñán AA. A contemporary study of iterative methods. Elsevier, New York. 2018.

[10] Argyros IK, Magreñán AA. Iterative methods and their dynamics with applications. CRC Press, New York, USA. 2017.

[11] Argyros IK, George S. Mathematical modeling for the solution of equations and systems of equations with applications. Nova Publishes, New York. 2020.

[12] Argyros IK, George S. On the complexity of extending the convergence region for Traub's method. Journal of Complexity. 2020; 56: 101423. Available from: https://doi.org/10.1016/j.jco.2019.101423.

[13] Jarratt P. Some fourth order multipoint iterative methods for solving equations. Mathematics of Computation. 1966; 
20: 434-437.

[14] Montazeri H, Soleymani F, Shateyi S, Motsa SS. On a new method for computing the numerical solution of systems of nonlinear equations. Journal of Applied Mathematics. 2012; 2012(SI06): 1-15. Available from: https:// doi.org/10.1155/2012/751975.

[15] Rheinboldt WC. An adaptive continuation process of solving systems of nonlinear equations. Banach Center Publications. 1978, 3: 129-142. Available from: https://eudml.org/doc/208686.

[16] Traub JF. Iterative methods for the solution of equations prentice Hall. New Jersey, USA. 1964.

[17] Vucheva V, Kolkovska N. Convergence analysis of finite difference scheme for sixth order Boussinesq equation. AIP Conference Proceedings. 2018; 1978: 470033. 